\title{
The Clinical Usefulness of Endoscopic Ultrasound-Guided Fine Needle Aspiration and Biopsy for Rectal and Perirectal Lesions
}

\author{
Jae Seung Soh, Ho-Su Lee, Seohyun Lee, Jungho Bae, Hyo Jeong Lee, Sang Hyoung Park, Dong-Hoon Yang, \\ Kyung-Jo Kim, Byong Duk Ye, Seung-Jae Myung, Suk-Kyun Yang, Jin-Ho Kim, Jeong-Sik Byeon \\ Department of Gastroenterology, Asan Medical Center, University of Ulsan College of Medicine, Seoul, Korea
}

\begin{abstract}
Background/Aims: Endoscopic ultrasound-guided fine needle aspiration and/or biopsy (EUS-FNA/B) have been used to diagnose subepithelial tumors (SETs) and extraluminal lesions in the gastrointestinal tract. Our group previously reported the usefulness of EUS-FNA/B for rectal and perirectal lesions. This study reports our expanded experience with EUS-FNA/B for rectal and perirectal lesions in terms of diagnostic accuracy and safety. We also included our new experience with EUS-FNB using the recently introduced ProCore needle. Methods: From April 2009 to March 2014, EUS-FNA/B for rectal and perirectal lesions was performed in 30 consecutive patients. We evaluated EUS-FNA/B performance by comparing histological diagnoses with final results. We also investigated factors affecting diagnostic accuracy. Results: Among 10 patients with SETs, EUS-FNA/ B specimen results revealed a gastrointestinal stromal tumor in 4 patients and malignant lymphoma in 1 patient. The diagnostic accuracy of EUS-FNA/B was 50\% for SETs (5/10). Among 20 patients with non-SET lesions, 8 patients were diagnosed with malignant disease and 7 were diagnosed with benign disease based on both EUS-FNA/B and the final results. The diagnostic accuracy of EUS-FNA/B for non-SET lesions was $75 \%(15 / 20)$. The size of lesions was the only factor related to diagnostic accuracy $(P=0.027)$. Two complications of mild fever and asymptomatic pneumoperitoneum occurred after EUS-FNA/B. Conclusions: The overall diagnostic accuracy of EUS-FNA/B for rectal and perirectal lesions was 67\% (20/30). EUS-FNA/B is a clinically useful method for cytological and histological diagnoses of rectal and perirectal lesions. (Intest Res 2015;13:135-144)
\end{abstract}

Key Words: Endoscopic ultrasound-guided fine needle aspiration; Biopsy, fine needle; Rectum; Perirectum

\section{INTRODUCTION}

Endoscopic ultrasound (EUS) has been used to examine abnormal lesions of the gastrointestinal tract and other organs. Notably, it has been used to evaluate subepithelial

Received September 19, 2014. Revised November 17, 2014.

Accepted December 9, 2014.

Correspondence to Jeong-Sik Byeon, Department of Gastroenterology,

Asan Medical Center, University of Ulsan College of Medicine, 88 Olympic-ro

43-gil, Songpa-gu, Seoul 138-736, Korea. Tel: +82-2-3010-3905, Fax: +82-

2-476-0824,E-mail: jsbyeon@amc.seoul.kr

Financial support: None. Conflict of interest: None. tumors (SETs) or extraluminal lesions compressed in the intestinal wall. EUS enables clinicians to measure the invasion depth of a tumor, to identify the origin of the wall layer of a SET, and to determine whether a lesion is intraluminal or extraluminal. ${ }^{1,2}$ However, histopathological specimens are usually necessary to confirm the diagnosis of a lesion. EUSguided aspiration or biopsy using fine needle devices have been used for this purpose. These methods allow clinicians to acquire appropriate tissue samples from subepithelial lesions that are not accompanied by mucosal changes or extraluminal lesions near the gastrointestinal wall, or to sample mucosal lesions that demand deep biopsy, such as recurrent or metastatic lesions.

\footnotetext{
๑ Copyright 2015. Korean Association for the Study of Intestinal Diseases. All rights reserved.

This is an Open Access article distributed under the terms of the Creative Commons Attribution Non-Commercial License (http://creativecommons.org/licenses/by-nc/3.0)

which permits unrestricted non-commercial use, distribution, and reproduction in any medium, provided the original work is properly cited.
} 
EUS-guided fine needle aspiration (EUS-FNA) has been established as a highly effective method for the pancreatic ${ }^{3}$ and upper gastrointestinal fields. ${ }^{4}$ Moreover, attempts to acquire core tissues for more accurate diagnostic assays have contributed to the development of EUS-guided fine needle biopsy (EUS-FNB) methods, such as Trucut or ProCore needle biopsy. ${ }^{5}$ Although EUS-guided procedures have also been performed in the lower gastrointestinal tract, they have been mostly confined to rectal or perirectal lesions because of poor accessibility of the EUS scope beyond the rectum. Consequentially, only a few studies of EUS-guided fine needle aspiration and/or biopsy (EUS-FNA/B) for lesions of the lower digestive tract have been reported. ${ }^{6-8}$

Our group previously reported a small, retrospective study that included 11 patients who underwent EUS-FNA and Trucut needle biopsy for rectal and perirectal lesions in $2011 .{ }^{8}$ In that study, these procedures were found to be useful in patients with rectal and perirectal lesions, and demonstrated an ability to provide accurate diagnoses in 10 of 11 patients. Subsequently, we have gained more experience with EUS-FNA/B for rectal and perirectal lesions, especially for EUS-guided ProCore needle biopsy, which was introduced in 2012. Therefore, the present study aimed to report our expanded experience with EUS-FNA/B for the diagnosis of rectal and perirectal lesions, focusing on diagnostic accuracy and safety, and included nine cases performed with a ProCore needle.

\section{METHODS}

\section{Patients}

From April 2009 to March 2014, EUS-FNA/B for rectal and perirectal lesions was performed in 30 consecutive patients at the Asan Medical Center, Seoul, Korea. Patients had masses or wall thickening in the rectum or perirectum that was detected by an imaging modality, such as abdominopelvic CT or MRI, and/or endoscopy. A presumptive diagnosis was made based on imaging and endoscopy findings before performing EUS-FNA/B. Because the prior imaging study or forceps biopsy using sigmoidoscopy or colonoscopy failed to make an accurate diagnosis, or could not be performed because of the location of the submucosal or extraluminal area, subsequent EUS-FNA/B procedures were recommended for these patients. EUS-FNB was performed in selected patients in whom an adequate tissue sample from initial EUSFNA could not be obtained or who required a larger sample with intact structural architecture for an accurate diagnosis.
All patients provided informed consent for EUS-FNA/B. Patients who had definite intraluminal cancer in the rectum were excluded from this study.

\section{EUS-FNA/B Techniques}

Prior to performing the EUS procedure, a detailed review of previous abdominopelvic imaging and/or endoscopy for rectal or perirectal lesions was carried out. Radial scanning EUS (GF-UM 2000; Olympus Co., Tokyo, Japan) was performed to examine the characteristics and location of the lesion. A linear-array echoendoscope (GF-UCT240-AL; Olympus Optical, Tokyo, Japan) was then inserted for aspiration or biopsy. EUS-FNA was performed using 19- or 22-gauge disposable needles (Echotip Ultra; Cook Medical Inc., Bloomington, IN, USA). After the FNA needle was advanced through the instrument channel of the linear-array echoendoscope, the internal stylet was removed and the needle was reciprocated back and forth within the lesion. The needle was withdrawn from the scope and air was infused with a syringe to extract the tissue material onto a slide. After confirming whether the tissue material was adequate, the needle was again passed through the lesion.

EUS-FNB was performed using two different methods: Trucut needle biopsy or ProCore needle biopsy. An EUSTrucut using a 19-gauge needle device (Quick-core; Cook Medical Inc.) fitted with a spring-loaded mechanism was the basic principle underlying the procedure. After the needle was placed in the lesion, the specimen tray was advanced into the lesion using a spring-loaded handle to obtain core tissue. An EUS-ProCore needle biopsy was newly introduced as a procedure in 2012. Thus, EUS-FNB with ProCore needle was not examined in our previous report ${ }^{8}$ and we newly analyzed the performance of EUS-FNB with ProCore needle in the present study. A fine needle biopsy was also performed using a 22-gauge needle device (Echotip ProCore-22; Cook Medical Inc.) with a reverse bevel design. The procedure with a ProCore needle biopsy was similar to the EUS-FNA technique using retrograde needle motion to acquire shearing tissues; however, the difference was that the needle for the ProCore biopsy was equipped with a 10-mL suction syringe after removing the stylet to extract core tissues. The specimens collected from all procedures were preserved in formalin for subsequent histopathological analysis. All patients underwent these procedures under conscious sedation with intravenously administered midazolam and meperidine. All procedures were performed by one of five faculty interventional endoscopists (Byeon JS, Ye BD, Yang 
DH, Park DH, or Kim DH).

\section{Outcome Parameters}

The primary outcome parameter was the percentage of patients in whom a correct histopathological diagnosis was made from tissue samples obtained by EUS-FNA/B. Diagnostic accuracy was defined as the proportion of patients in which the histopathological diagnoses of EUS-FNA/B was consistent with the final results. The final results were established according to the diagnosis from resected specimens in patients who underwent surgery. In patients who did not undergo surgery, the final results were made based on the long-term clinical course along with the EUS-FNA/B result and imaging studies during the follow-up period.

The secondary outcome parameters were the rates of optimal sampling, and the occurrence of complications. Additionally, medical records regarding the type of EUS-FNA/ $B$ procedure and the number of passes required to obtain samples were reviewed. Optimal samples were defined as tissue samples with high cellularity and quality that enabled histopathological diagnoses.

Finally, we investigated factors affecting diagnostic accuracy by analyzing the relationship between the diagnostic yield of EUS-FNA/B and factors such as age, sex, size of lesion, procedure methods (FNA or FNB), ProCore use, needle passes, EUS echo pattern (heterogeneous or homogenous), and location of lesions (rectal or perirectal).

This study protocol was approved by the Institutional Review Board of the Asan Medical Center, Seoul, Korea (protocol no. 2014-0923).

\section{Statistical Analysis}

The chi-square test or Fisher's exact test was used to evaluate the factors related to diagnostic accuracy. Data analysis was performed using SPSS 20.0 (SPSS, Chicago, IL, USA). $P$ values $<0.05$ were considered statistically significant.

\section{RESULTS}

\section{Patients}

The median age of the 30 patients who underwent EUSFNA/B was 56 years (range, 33-81 years), and 18 patients were male. Additionally, 18 patients had lesions in the rectum and 12 patients had lesions in the perirectal area. Of the 18 patients with rectal lesions, 10 were cases of SET. The pre- senting symptoms were as follows: 5 patients with melena or hematochezia, 3 patients with anal pain or a palpable anal lesion, 3 patients with abdominal pain and fever, 2 patients with bowel habit changes, and 1 patient with hematuria. The remaining 16 patients did not have any symptoms at the time of their hospital visit, and most of them were referred by primary clinics or other departments because of abnormal findings from an imaging study or colonoscopy. Half of the patients (15/30) had a history of malignant disease, including colorectal cancer in 7 patients, gastric cancer in 5 patients, lymphoma in 1 patient, leukemia in 1 patient, and malignant gastrointestinal stromal tumor (GIST) in 1 patient.

Most patients underwent colonoscopy or sigmoidoscopy, except for 3 patients with perirectal lesions. Endoscopic forceps biopsy was performed for mass-forming elevated lesions in 10 patients; however, all patients showed non-diagnostic findings such as non-specific inflammation. All patients revealed abnormal findings in CT or MRI analyses. The final results were confirmed by surgical specimens in 8 patients, histopathological findings of EUS-FNA/B that indicated malignancy in 9 patients, and clinical courses with imaging studies during the follow-up period in 13 patients. We carried out EUS-FNA alone in 9 patients, EUS-FNB alone in 12 patients, and both EUS-FNA and FNB in 9 patients (Table 1).

\section{EUS-FNA/B Results and Diagnostic Accuracy in SETs}

A presumptive diagnosis of SETs, based on the findings of imaging studies and colonoscopy, was made for 10 patients. The median size of SETs was $25 \mathrm{~mm}$ (range, 11-56 mm). The presumptive diagnoses, location, size, EUS procedure, histopathological diagnoses of EUS-FNA/B, treatment, follow-up time (in months), and final results are summarized in Table 1. The findings of EUS in all SETs showed hypoechoic masses, and EUS procedures were successfully completed in all cases. EUS-FNB was performed in 8 of 10 patients; including EUS-FNB alone in give patients (Trucut in 2 cases and ProCore in 3 cases), and both EUS-FNA and FNB in three patients. Among the 3 cases of both EUS-FNA and FNB, 2 underwent EUS-FNA and FNB at the same time, while 1 patient (no. 5) initially underwent EUS-FNA, but histopathological analysis confirmed nonspecific inflammation. Because the findings of imaging studies for the latter patient strongly suggested a malignancy, another EUS-FNA and Trucut needle biopsy was subsequently attempted. Consequently, malignant lymphoma was diagnosed. Only 2 patients underwent EUS-FNA alone. A median of 3 FNA needle passes (range, 2-4) and a median of 2.5 FNB needle 


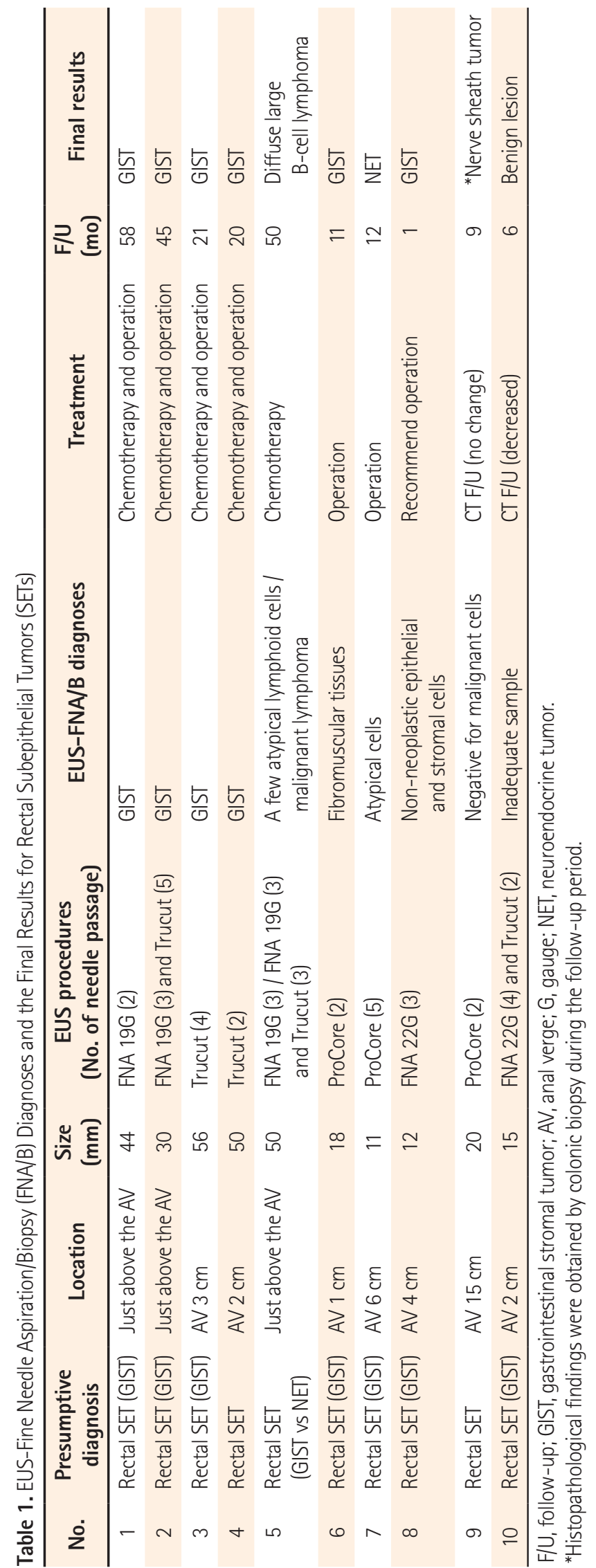

passes (range, 2-5) were carried out in SET cases.

The results of the EUS-FNA/B specimens in SETs were as follows: GIST in four patients, malignant lymphoma in 1 patient, and various benign results in 4 patients. An inadequate tissue sample was obtained for 1 patient (no. 10). GIST was diagnosed as spindle cells based on immunohistochemical (IHC) staining of EUS-FNA/B specimens (Fig. 1). There were 4 patients who showed only benign cells and tissues by EUSFNA/B who could not be diagnosed with any definite disease. Thus, surgical treatment was recommended for them because the size of the SETs was nearly $2 \mathrm{~cm}$, and we could not exclude the potential for malignancy. Among these 4 patients, 2 were diagnosed with GIST and neuroendocrine tumors based on analyses of the surgical specimen. Eventually, 6 patients were diagnosed with GIST, and 1 patient each was diagnosed with diffuse large B cell lymphoma, neuroendocrine tumor, and nerve sheath tumor. In the remaining patient, the final results were considered to be a benign lesion based on the clinical course and follow-up imaging studies. The median follow-up period of patients with SETs was 16 months (range, 1-58) and that of patients who did not undergo operation or chemotherapy (no. 8-10) was 6 months (range, 1-9). The diagnostic accuracy of EUS-FNA/B based on the final results was $50 \%(5 / 10)$ (Table 2). When we analyzed diagnostic accuracy according to the size of lesions, the diagnostic yield for SETs less than $20 \mathrm{~mm}$ in size was $0 \%$ $(0 / 4)$ and that for SETs of $20 \mathrm{~mm}$ in size or larger was $83.3 \%$ $(5 / 6)$.

\section{EUS-FNA/B Results and Diagnostic Accuracy in Non-SET Lesions}

After excluding 10 patients with SETs, a total of 20 patients underwent EUS-FNA/B for their rectal or perirectal lesions that were detected by an imaging study or colonoscopy. The median size of non-SET lesions was $28 \mathrm{~mm}$ (range, 13-60 $\mathrm{mm})$. Among the 20 patients, 13 had a history of cancer, including 7 cases of colorectal cancer, 3 cases of advanced gastric cancer, and 1 case each of Hodgkin's lymphoma, acute lymphocytic leukemia, and small bowel GIST. Additionally, 16 patients (80\%) had a primary, recurrent, or metastatic malignancy as a presumptive diagnosis. The other four patients underwent EUS-FNA/B for lesions that were suspected to be perirectal abscesses or endometriosis. Details, including the histopathological diagnoses of EUS-FNA/B and the final results of non-SET lesions, are shown in Table 3.

Most patients in the non-SET lesion group showed a hypoechoic mass or nodule with heterogeneity in EUS. One 

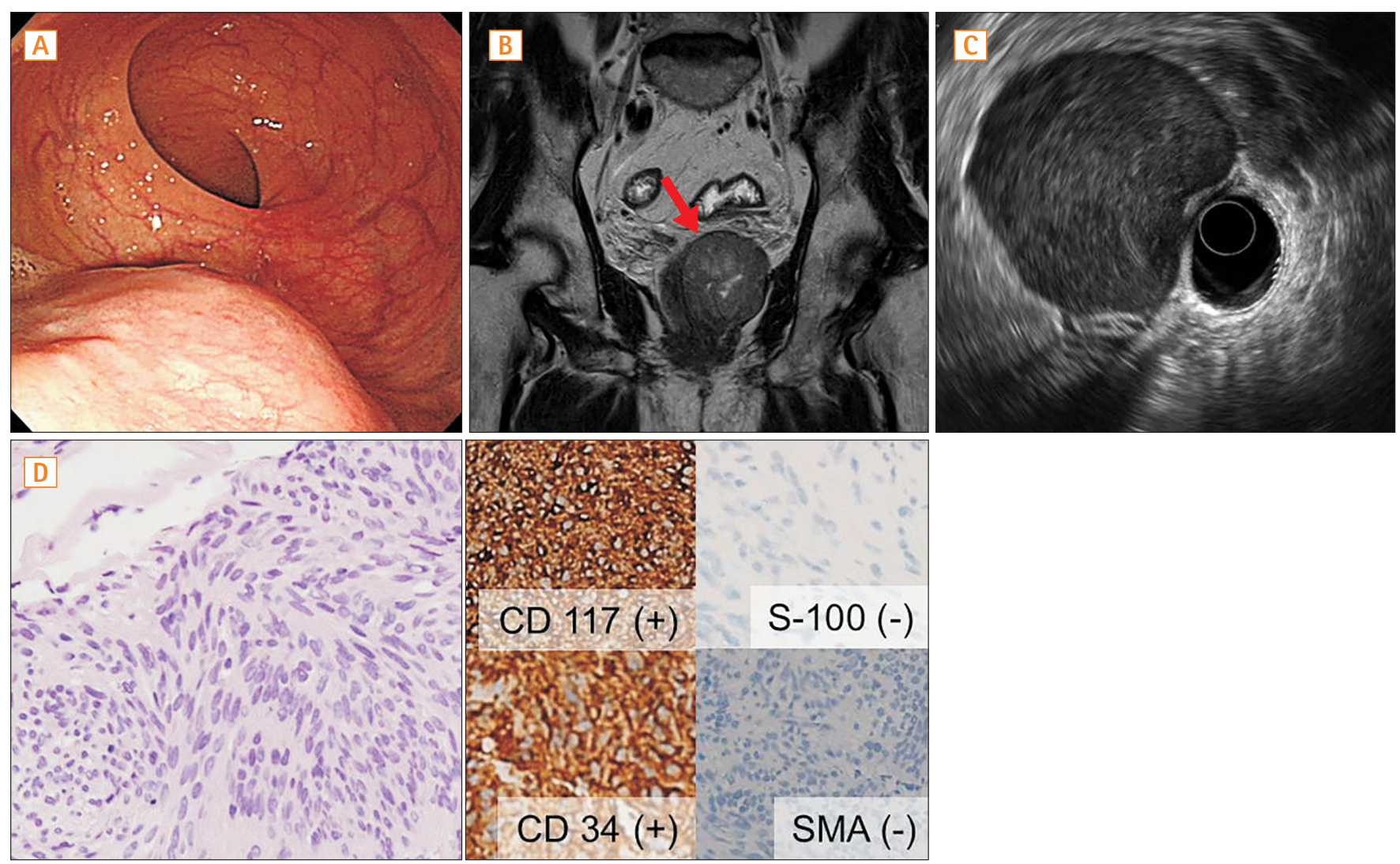

Fig. 1. A 61-year-old man (no. 4). (A) Colonoscopy showing a subepithelial tumor $\sim 3 \mathrm{~cm}$ in diameter in the distal rectum. (B) MRI scan showing a welldefined mass with an internal high-density component $\sim 6 \mathrm{~cm}$ in diameter in the distal rectum. (C) EUS showing a well-demarcated hypoechoic mass with central heterogeneous echogenicity approximately $5.0 \times 4.3 \mathrm{~cm}$ in size in the rectum, which displaced the prostate. (D) Histological examination of the EUS-fine needle biopsy specimen showing spindle cells $(\mathrm{HEE}, \times 200)$ that were positive for CD117 and CD34, but negative for smooth muscle actin and S-100 by immunohistochemistry ( $\times 200)$. This profile was diagnostic of a gastrointestinal stromal tumor. SMA, smooth-muscle actin.

Table 2. Diagnostic Accuracy of EUS-Fine Needle Aspiration/Biopsy (FNA/B) for Rectal Subepithelial Tumors (SETs) and Non-SET Rectal or Perirectal Lesions

\begin{tabular}{|c|c|c|c|c|c|c|c|c|c|}
\hline \multirow[b]{2}{*}{ Diagnostic category } & \multicolumn{5}{|c|}{ SETs } & \multicolumn{3}{|c|}{ Non-SET lesions } & \multirow[b]{2}{*}{ Total of all lesions } \\
\hline & GIST & Lymphoma & NET & $\begin{array}{c}\text { Other } \\
\text { benign SETs }\end{array}$ & Total & Malignancy & Benign & Total & \\
\hline Diagnostic & 4 & 1 & 0 & 0 & $5(50.0)$ & 8 & 7 & $15(75.0)$ & $20(66.7)$ \\
\hline Non-diagnostic & 2 & 0 & 1 & 1 & $4(40.0)$ & 4 & 0 & $4(20.0)$ & $8(26.7)$ \\
\hline Inadequate sample & 0 & 0 & 0 & 1 & $1(10.0)$ & 0 & 1 & $1(5.0)$ & $2(6.7)$ \\
\hline
\end{tabular}

Values are presented as $n$ or $n(\%)$.

SET, subepithelial tumor; GIST, gastrointestinal stromal tumor; NET, neuroendocrine tumor.

patient (no. 27) showed a diffuse hypoechoic thickened wall. EUS-FNB was performed in 12 of 20 patients, including EUSFNB alone in eight patients and both EUS-FNA and FNB in four patients. For the four patients who underwent both EUS-FNA and FNB, two underwent EUS-FNA and FNB at the same time, while two (no. 16 and 28) suspected of malignancies subsequently underwent EUS-FNA and FNB. EUS-
FNA alone was performed in 8 patients. A median of three FNA needle passes (range, 2-5) and a median of three FNB needle passes (range, 2-7) were performed.

A total of 8 patients showed malignant cells in their EUSFNA/B specimens. Recurrent Hodgkin's lymphoma was confirmed based on histopathological analyses with IHC staining by EUS-FNB in one patient (no. 16; Fig. 2), while 11 


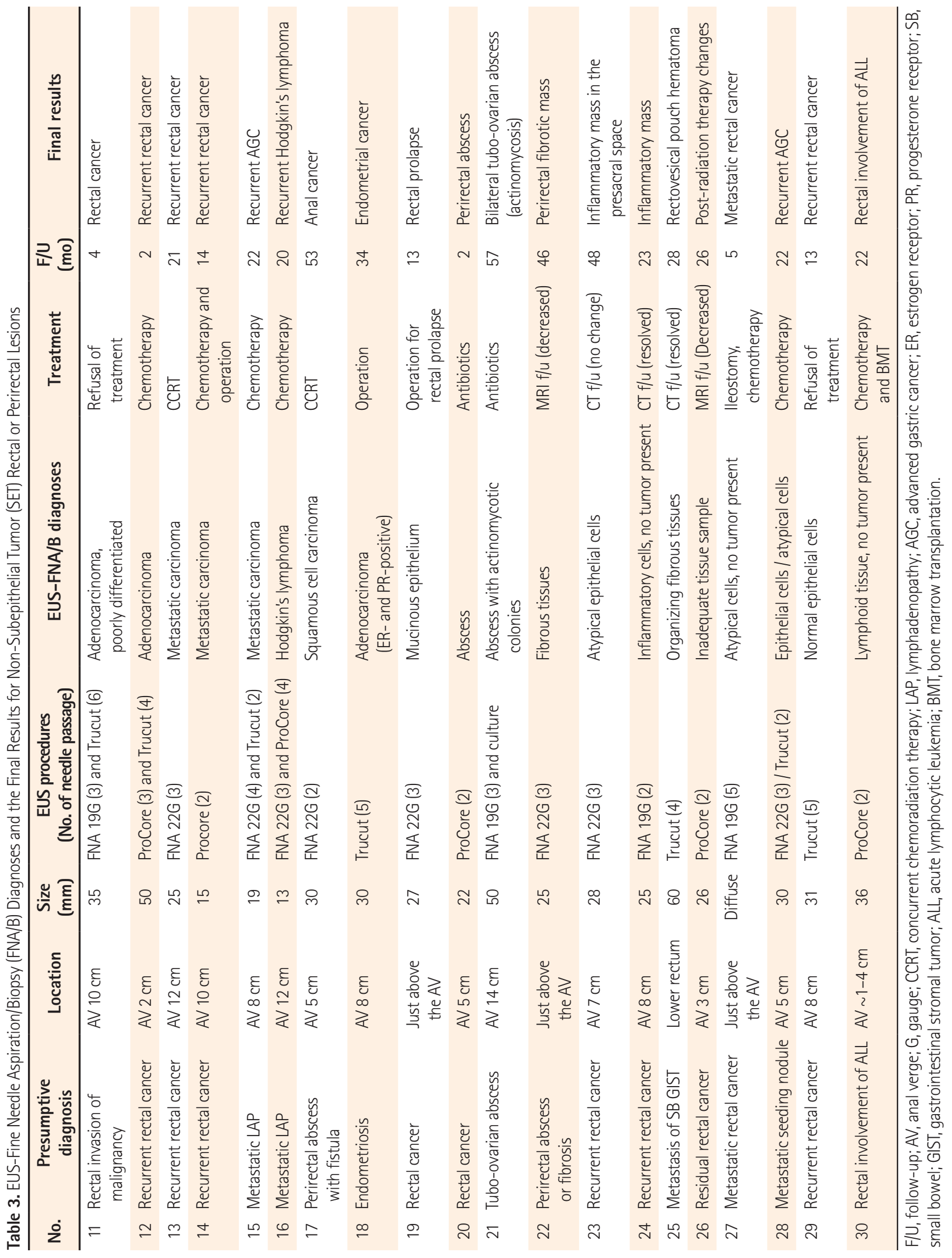



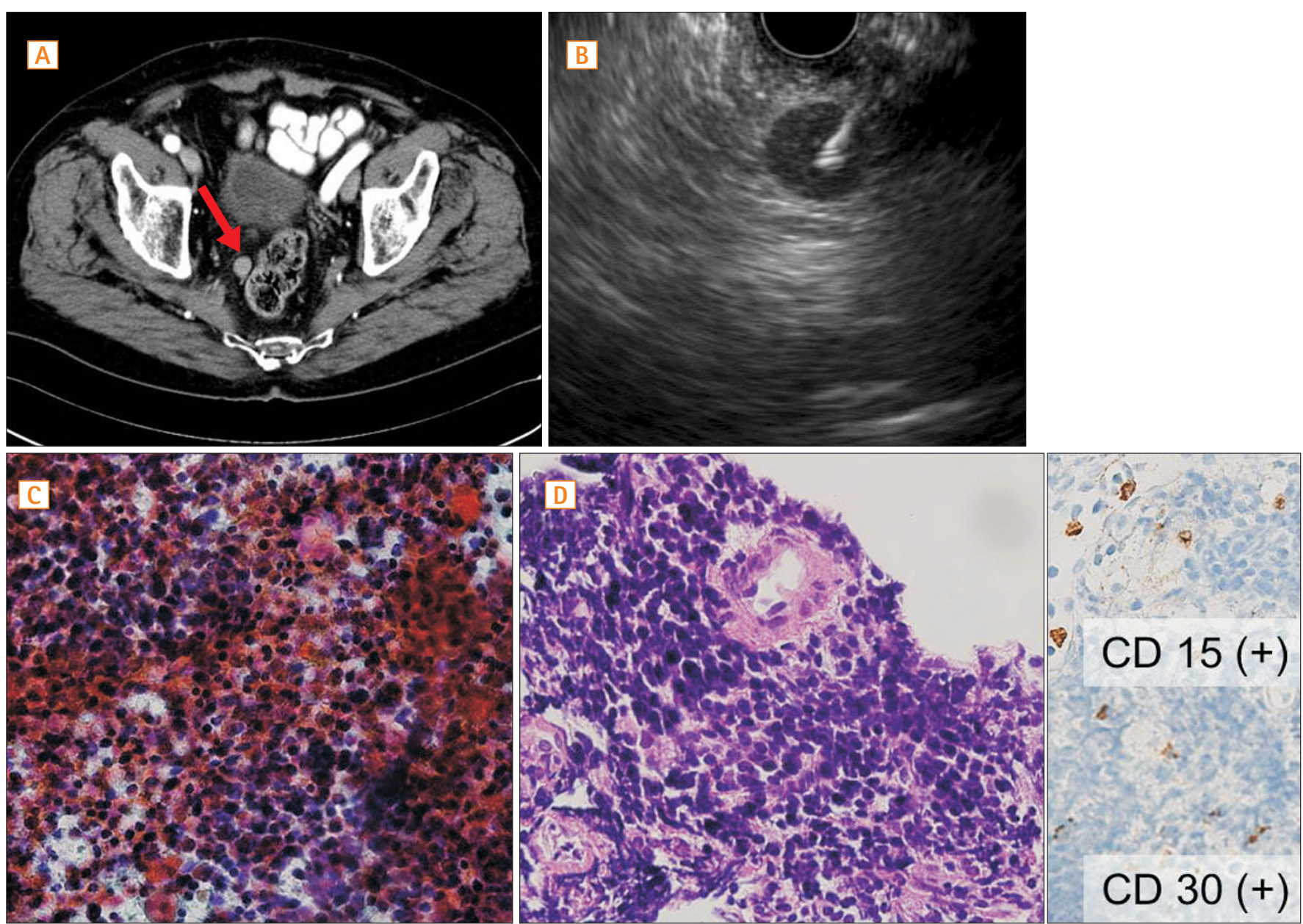

Fig. 2. An 81-year-old man (no. 16) with a history of Hodgkin's lymphoma. (A) CT showing a well-defined nodule $\sim 1 \mathrm{~cm}$ in diameter near the upper rectum. (B) Linear EUS showing a round hypoechoic lymph node $\sim 1.2 \mathrm{~cm}$ in diameter located near the upper rectum. The 22-gauge ProCore needle was inserted into the lesion. (C) Histological examination of a sample obtained upon the first EUS-fine needle aspiration using a 22-gauge aspiration needle showed atypical cells $\left(H \& E E_{1} \times 200\right)$ but was insufficient for a definitive diagnosis. (D) Histological examination of a specimen obtained upon the second EUS-fine needle biopsy using 22-gauge ProCore needle showing atypical lymphoid cells (HEE, $\times 200)$ that were positive for CD30 and CD15 by immunohistochemistry (×200). This profile was diagnostic of recurrent Hodgkin's lymphoma.

patients were diagnosed with benign diseases by EUS-FNA/ B. For five patients, a malignant or recurrent disease was suggested as a presumptive diagnosis, but inflammatory cells or fibrotic tissues were detected in the EUS-FNA/B specimens. Consequently, they were finally diagnosed with benign diseases. The final results were as follows: recurrent malignancy in 8 patients, primary cancer in 4 patients, inflammatory or fibrotic mass in 4 patients, abscess in 2 patients, hematoma in 1 patient, and rectal prolapse in 1 patient. Among the 12 patients with final results of malignant disease, malignant cells were not detected in the EUS-FNA/B specimen in 4 patients. Additionally, inadequate tissue samples for diagnosis were obtained during the EUS-procedure in 1 patient (no. 26). The median follow-up period of patients with non-
SET lesions was 22 months (range, 2-57) and that of patients who were diagnosed with benign lesions in the final results (no. 19-26) was 27 months (range, 2-57). EUS-FNA/ $\mathrm{B}$ diagnoses were consistent with the final results in 15 of 20 patients and the diagnostic accuracy of EUS-FNA/B in nonSET lesions was $75 \%$ (Table 2).

\section{Factors Related to Diagnostic Accuracy}

The size of lesions was the only factor related to diagnostic accuracy $(P=0.027)$. Other factors including age, sex, procedure methods, ProCore use, needle passes, heterogeneous echo, and rectal location were not statistically significant (Table 4). 
Table 4. Factors Related to Diagnostic Accuracy for Rectal Subepithelial Tumor (SET) and Non-SET Rectal or Perirectal Lesions

\begin{tabular}{lccc}
\hline \multicolumn{1}{c}{ Factors } & $\begin{array}{c}\text { Diagnostic } \\
(\mathbf{n}=\mathbf{2 0})\end{array}$ & $\begin{array}{c}\text { Non-diagnostic* } \\
(\mathbf{n}=10)\end{array}$ & P-value \\
\hline Median age, yr (range) & $61.5(33-81)$ & $54.0(38-69)$ & 0.320 \\
Male gender & $11(55.0)$ & $7(70.0)$ & 0.694 \\
Size, mm (range) & $34.2(13-60)$ & $22.1(11-36)$ & 0.027 \\
SETs & $5(25.0)$ & $5(50.0)$ & 0.171 \\
Procedure & & & 0.419 \\
$\quad$ EUS-FNA & $8(40.0)$ & $2(20.0)$ & \\
$\quad$ EUS-FNB & $7(35.0)$ & $6(60.0)$ & \\
$\quad$ EUS-FNA and FNB & $5(25.0)$ & $2(20.0)$ & \\
ProCore & $4(20.0)$ & $5(50.0)$ & 0.091 \\
Needle passes, times & $4.2(2.3,2-6)$ & $3.7(1.6,2-9)$ & 0.580 \\
(SD, range) & & & \\
Heterogeneous echo & $18(90.0)$ & $7(70.0)$ & 0.300 \\
Rectal lesions & $11(55.0)$ & $7(70.0)$ & 0.694 \\
\hline
\end{tabular}

Values are presented as $n$ (\%) or median (range).

*Nondiagnostic included patients with inadequate sample.

FNA, fine needle aspiration; FNB, fine needle biopsy.

\section{Complications}

In 2 cases, complications were noted after EUS-FNA/B. In 1 patient (no. 11), fever occurred 1 day after EUS-FNA/ B. Antibiotics were infused intravenously and she was uneventfully discharged 4 days after EUS-FNA/B. Prophylactic antibiotics had not been administered before her EUS-FNA/ B procedure. Another patient (no. 14) was diagnosed with a pneumoperitoneum radiologically after EUS-FNB. However, he did not show any symptoms associated with peritonitis and was discharged two days later. A total of 22 patients $(73 \%)$ received prophylactic antibiotics prior to their EUS procedure.

\section{DISCUSSION}

Here, we demonstrate that EUS-FNA/B allowed us to make a correct diagnosis in 20 of 30 patients $(66.7 \%)$ with rectal SETs or non-SET rectal and perirectal lesions. Table 2 shows the diagnostic accuracy of EUS-FNA/B for rectal SETs and non-SET rectal and perirectal lesions. Although this rate does not appear to be very high, EUS-FNA/B has its own diagnostic strengths as it can help us in making treatment decisions in patients with rectal and perirectal lesions for which it is difficult to decide on a management plan.
Previous studies of EUS-FNA performed in the lower gastrointestinal tract showed a relatively high diagnostic accuracy. Hara et al. ${ }^{6}$ reported that the diagnostic accuracy of EUS-FNA was $90 \%$ in 10 patients with rectal and sigmoid lesions, including rectal cancer, endometriosis, and GIST. Sasaki et al. ${ }^{7}$ found that the diagnostic yield of EUS-FNA was $95.5 \%$ (21 of 22) in submucosal and extrinsic masses of the colon and rectum. We do not know why our diagnostic value was so low. However, when we considered non-SET lesions separately, the diagnostic accuracy was $75 \%$, which was quite good. Many previous studies have reported that EUS-FNA has a relatively good accuracy ( $80 \%$ to $89 \%$ ) in diagnosing SETs of the gastrointestinal tract. ${ }^{9-11}$ Compared to previous studies, our present study showed a relatively low accuracy (50\%) for EUS-FNA/B in SETs. The reason why the diagnostic accuracy was different between SETs and nonSET lesions is not clear. Because the diagnostic accuracy was lower in small lesions, a high proportion of lesions less than $20 \mathrm{~mm}(40 \%, 4 / 10)$ in SETs may be a cause of low diagnostic accuracy in SETs. In comparison, the proportion of lesions less than $20 \mathrm{~mm}$ was only $15 \%(3 / 20)$ in non-SET lesions for which a higher diagnostic accuracy of EUS-FNA/B was achieved than that for SETs. Further studies are necessary to explain why the diagnostic accuracy is different between SETs and non-SETs.

In our previous study, we reported the usefulness of EUSFNA and Trucut biopsy in rectal or perirectal lesions, demonstrating high diagnostic accuracy (91.7\%) and safety. ${ }^{8} \mathrm{In}$ addition to these findings, there were some new findings in our current study. First, we identified that the size of lesions was significantly related to the diagnostic accuracy of EUSFNA/B $(P=0.027)$. Diagnostic yield was lower in SETs less than $20 \mathrm{~mm}$ than in those over than $20 \mathrm{~mm}(0 \%$ vs. 83.3\%). This finding suggests that EUS-FNA/B in small lesions may need further improvement for the diagnostic usefulness. Sufficient training of clinicians for EUS-FNA/B procedures and strategic experience with large to small lesions may lead to the desired improvement in diagnostic yield, especially for small lesions. Second, our current study contained nine patients who underwent EUS-FNB using ProCore needle, which is a new FNB needle and was not used in our previous study. Trucut biopsy had procedural difficulties such as needle stiffness and misfiring, which might lead to technical failure. $^{12}$ The ProCore needle was developed to overcome these weak aspects of the Trucut needle. Because ProCore needles have an advantage of relatively easy acquisition of core tissues with structural preservation, the number of needle passages needed to obtain adequate tissue specimens can be 
reduced. Witt et al. ${ }^{13}$ reported that ProCore needles required fewer passes to acquire specimens compared to EUS-FNA (2.11 vs. 2.94). In the current study, the mean number of needle passes of ProCore needles, Trucut needles, and FNA were $2.67,3.67$, and 3.06 , respectively. The number of needle passes needed with ProCore needles was less than for other procedures. Despite the advantage regarding needle passes needed, the diagnostic accuracy of EUS-FNB using ProCore needles $(44.4 \%, 4 / 9)$ was not adequate in the current study. However, EUS-guided ProCore needle biopsy has shown a similar diagnostic efficacy to EUS-FNA in other comparative studies. ${ }^{14,15}$ Thus, we suggest that further studies are necessary to confirm the usefulness of EUS-FNB using ProCore needles in terms of both diagnostic accuracy and the number of needle passes needed.

Concurrent or subsequent harvesting of tissue samples using both EUS-FNA and FNB has been reported to enable a higher diagnostic accuracy compared to performing each procedure separately. ${ }^{16,17}$ In the current study, both EUS-FNA and FNB were performed simultaneously in seven patients to obtain more suitable tissue samples for architectural preservation with IHC staining. We obtained proper specimens for diagnosis in five cases. For one patient (no. 16; Fig. 2) who had a past history of Hodgkin's lymphoma, we could obtain a confirmative diagnosis of recurrent lymphoma through subsequent EUS-FNB after achieving an insufficient histopathological result by EUS-FNA. Undoubtedly, with architectural examination and IHC staining, the diagnostic accuracy could be improved. Therefore, EUS-FNB could be used as a complimentary procedure to overcome the shortcomings of EUS-FNA, and even used concurrently or subsequently with EUS-FNA and FNB.

When comparing the presumptive diagnosis before EUSFNA/B to the final diagnosis in 20 non-SET lesions, the diagnoses were coincident in 12 patients. Among the remaining eight patients, the presumptive diagnosis in seven patients was changed or corrected based on the histopathological results of EUS-FNA/B. In two of seven patients (no. 17 and 18), the lesions were thought to be benign, but were later diagnosed as malignancies by EUS-FNA/B. In contrast, five patients (no. 19, 20, 23, 24, and 25) considered to have malignancies turned out to have benign lesions based on the histopathological results of EUS-FNA/B. Histopathological diagnoses via EUS-FNA/B could allow patients with benign rectal or perirectal lesions to avoid unnecessary operations. Additionally, EUS-FNA/B enabled patients with a malignancy to obtain a rapid diagnosis and proper treatment.

In the current study, EUS-FNA/B was safe to use, with only two complications of mild fever and asymptomatic pneumoperitoneum immediately following the procedures. No patient experienced any severe complication. These results are in agreement with previous studies that reported the safety of EUS-FNA/B with a low complication profile $(1-2 \%){ }^{18,19}$ In a recently published study, Levy et al. $^{20}$ reported that adverse events developed in $20.5 \%$ of cases, although serious adverse events only occurred in 5.6\% after EUS-FNA procedures in the lower gastrointestinal tract. In that study, bleeding and pain were the most common adverse events. Generally, the use of adequate prophylactic antibiotics, the proper selection of needle and passage number, and practice by an experienced operator can prevent these complications and adverse events of EUS-FNA/B in the rectum and perirectum.

Our study had several limitations. First, the analysis was retrospective and could have included biases as a consequence of unrecognized or unmeasured factors. Second, there was no on-site cytopathologist in our endoscopy unit, which might have been related to the low diagnostic accuracy. Third, this was a small-sized, single center study.

In conclusion, although our current findings showed only moderate diagnostic accuracy, EUS-FNA/B was observed to be a clinically useful method for performing cytological and histological examinations, including IHC staining, for rectal or perirectal lesions. A future large-scale, multi-center study is warranted.

\section{ACKNOWLEDGEMENTS}

We thank Professors Do Hoon Kim and Do Hyun Park for their contributions to the EUS-FNA/B procedures.

\section{REFERENCES}

1. Godfrey EM, Rushbrook SM, Carroll NR. Endoscopic ultrasound: a review of current diagnostic and therapeutic applications. Postgrad Med J 2010;86:346-353.

2. Zhu Q, Xu C, Hu D. Dose endoscopic ultrasonography impact diagnosis of intestinal diseases? Intest Res 2011;9:179-188.

3. Soetikno RM, Chang K. Endoscopic ultrasound-guided diagnosis and therapy in pancreatic disease. Gastrointest Endosc Clin N Am 1998;8:237-247.

4. Südhoff T, Hollerbach S, Wilhelms I, et al. Clinical utility of EUS FNA in upper gastrointestinal and mediastinal disease. Dtsch Med Wochenschr 2004;129:2227-2232.

5. Hucl T, Wee E, Anuradha S, et al. Feasibility and efficiency of a new $22 \mathrm{G}$ core needle: a prospective comparison study. Endoscopy 2013;45:792-798. 
6. Hara K, Yamao K, Ohashi K, et al. Endoscopic ultrasonography and endoscopic ultrasound-guided fine-needle aspiration biopsy for the diagnosis of lower digestive tract disease. Endoscopy 2003;35:966-969.

7. Sasaki Y, Niwa Y, Hirooka Y, et al. The use of endoscopic ultrasound-guided fine-needle aspiration for investigation of submucosal and extrinsic masses of the colon and rectum. Endoscopy 2005;37:154-160.

8. Boo SJ, Byeon JS, Park do H, et al. EUS-guided fine needle aspiration and trucut needle biopsy for examination of rectal and perirectal lesions. Scand J Gastroenterol 2011;46:1510-1518.

9. Arantes V, Logrono R, Faruqi S, Ahmed I, Waxman I, Bhutani MS. Endoscopic sonographically guided fine-needle aspiration yield in submucosal tumors of the gastrointestinal tract. J Ultrasound Med 2004;23:1141-1150.

10. Vander Noot MR, 3rd, Eloubeidi MA, Chen VK, et al. Diagnosis of gastrointestinal tract lesions by endoscopic ultrasoundguided fine-needle aspiration biopsy. Cancer 2004;102:157-163.

11. Hunt GC, Smith PP, Faigel DO. Yield of tissue sampling for submucosal lesions evaluated by EUS. Gastrointest Endosc 2003;57:68-72.

12. Strand DS, Jeffus SK, Sauer BG, Wang AY, Stelow EB, Shami VM. EUS guided 22 gauge fine needle aspiration versus core biopsy needle in the evaluation of solid pancreatic neoplasms. Diagn Cytopathol 2014;42:751-758.

13. Witt BL, Adler DG, Hilden K, Layfield LJ. A comparative needle study: EUS-FNA procedures using the HD ProCore ${ }^{\mathrm{TM}}$ and EchoTip $^{\circledR}$ 22-gauge needle types. Diagn Cytopathol 2013;41:10691074
14. Iglesias-Garcia J, Poley JW, Larghi A, et al. Feasibility and yield of a new EUS histology needle: results from a multicenter, pooled, cohort study. Gastrointest Endosc 2011;73:1189-1196.

15. Kim GH, Cho YK, Kim EY, et al. Comparison of 22-gauge aspiration needle with 22-gauge biopsy needle in endoscopic ultrasonography-guided subepithelial tumor sampling. Scand J Gastroenterol 2014;49:347-354.

16. Ginès A, Wiersema MJ, Clain JE, Pochron NL, Rajan E, Levy MJ. Prospective study of a Trucut needle for performing EUS-guided biopsy with EUS-guided FNA rescue. Gastrointest Endosc 2005;62:597-601.

17. Storch I, Jorda M, Thurer R, et al. Advantage of EUS Trucut biopsy combined with fine-needle aspiration without immediate on-site cytopathologic examination. Gastrointest Endosc 2006;64:505-511.

18. Eloubeidi MA, Tamhane A. Prospective assessment of diagnostic utility and complications of endoscopic ultrasound-guided fine needle aspiration. Results from a newly developed academic endoscopic ultrasound program. Dig Dis 2008;26:356363.

19. Thomas T, Kaye PV, Ragunath K, Aithal G. Efficacy, safety, and predictive factors for a positive yield of EUS-guided Trucut biopsy: a large tertiary referral center experience. Am J Gastroenterol 2009;104:584-591.

20. Levy MJ, Abu Dayyeh BK, Fujii LL, et al. Prospective evaluation of adverse events following lower gastrointestinal tract EUS FNA. Am J Gastroenterol 2014;109:676-685. 